\title{
CONSTRAINED NUCLEAR DESIGN
}

by

W. P. Barthold

NOTICE

This was prepured as an accuunt of work nnuent, Neither sponsored by the Unired sir emplayees, nor any of

Con

their contros, express or implied, or assumes any

makes any we responsibility for the accuracy, com-

legal liablity or

pleteness or usefulness of any infortesents that its we

product or prosess disclosed, or represents

would not infringe privately owned rights.

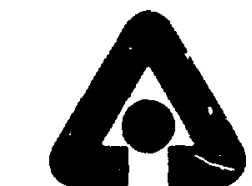

For Presentation At:

American Nuclear Society Topical Meeting,

"Advanced Reactors; Physics, Design and Esonomics," Atlanta, Georgia, September 8-11, 1974

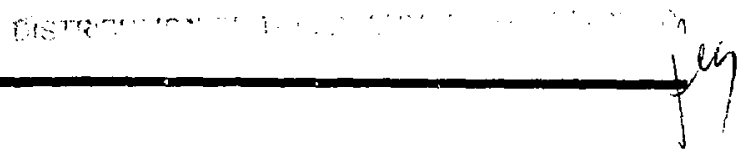




\author{
CONSTRAINED NUCLEAR DESIGN* \\ W. P. Barthold \\ Argonne National Laboratory, Argonne, Illinois
}

\begin{abstract}
By imposing constraints on the design of a reactor it is possible to reduce the number of iterations between mechanical, thermal and nuclear design. The constraints discussed are geometry constraints, physics constraints, pressure drop constraint, sodium velocity constraint and burnup and fluence limit constraints. In addition to reducing the number of design iterations, this approach helps to identify key design parameters as well as key design constraints.
\end{abstract}

\title{
I. INTRODUCTION
}

The final design of a reactor is the result of numerous iterations between thermal, mechanical and nuciear design. Design parameters like fuel pin diameter, pitch-to-diameter ratio, core helght, assembly size, etc., as well as core configuration and core dimension are belng finalized in this iteration process. The complexity of this process, its sequential nature and its duration provide enough incentive to search for a shortening of this process. The most obvious and best solution is a good first design which does not require major modifications to make it optimum. This goal is achieved by constraining some of the design parameters.

Various approaches in constraining the nuclear design are being used. They differ in the number of constraints applied, 1,e., the degree by which our existing knowledge of FBRs is being utilized.

Imposing constraints on the design does not mean the introduction of artificial boundaries for design parameters but recognizing that several of the design parameters are correlated and subject to upper or lower bounds. The use of single-stage pumps imposes constraints and so does a maximum burnup linit. There are bounds for the maximum sodium velocity as well as assembly size. The length of the operating cycle acts as restraint as well as the shape of the fuel assembly and the maximum linear heat rating.

\section{NUCLEAR DESIGN METHODS}

There are basically four methods which are being utilized in fast reactor design. They are briefly characterized in Table I. The first method uses the power density as input variable. The core size is constrained by the power output. The L/D ratio is varied and the performance

*Work performed under the auspices of the U. S. Atomic Energy Commission.

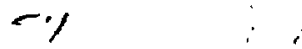


Table I. Fast Reartor Design Methods

INPUT

CONSTRAINT

Power Density

$\mathrm{kW} / \mathrm{ft}$

$\mathrm{kW} / \mathrm{ft}$

$\mathrm{kW} / \mathrm{ft}$

\author{
Power Output \\ Power Output \\ Power Output, Hexagonal Arrangement \\ Power Output, Hexagonal Arrangement, \\ Maximum Sodlum Velocity, Coolant \\ Pressure Drop, Burnup
}

of the reactor is evaluated. Th1s approach neglects any structure inside the core. Different fuel volume fractions have to be interpreted as different fuel pin diameters with certain pitch-to-diameter ratios. Fuel cycle cost calculations are rather restricted in their value because fabrication and processing costs used represent average costs only. Studies of this $\mathrm{kind}$ are well suited to analyze homogeneous reactors and to evaluate the potential of a heterogeneous reactor concept in terms of nuclear performance. However, any other conclusion has to be doubtful.

The second method eliminates some of the disadvantages of the first method. By defining a certain linear heat rate as input variable the structure of the fuel assembly is taken into account. The design is constrained by the power output. There is an unequivocal relation between core height and core radius. To each arbitrarily chosen core height belongs a core radius which defines the proper core size subject to the power output constraint. This method is very common among reactor designers. Fabrication cost calculations can be based on actual fuel element designs rather than on "reasonable" cost information for an "average assembly". Although this method rep":esents a significant improvement over the first method, it does not restrict the number of cases to be studied for core optimizations. One of the disadvantages of this method is the neglect of the finite size of the fuel assembly. The interpretation of results obtained by this method in terms of actual core layouts is rendered more difficult.

This leads to the third method which takes into account the size of the fuel assembly. In addition to this constraint, an either hexagonal arrangement of fuel assemblies or an arrangement which shows a circular periphery is being postulated to avoid unnecessary lot spots. This method deals with real core layouts. For a given power output, there are only a few possible core arrangements.

Method four goes further by imposing two more constraints: pressure drop and maximum sodium velocity. With these additional constraints the number of core layouts can be reduced to very few arrangements with only one degree of freedom: the fuel rod diameter. But by adding the burnup limit to the other constraints even the fuel pin diameter is not a free variable any more. 
The definition of nuclear design constraints is based on our knowledge of fast reactors in general and mechanical, thermal and nuclear design of these reactors in particular. In the following five nuclear design constraints will be discussed.

\section{(a) geometry constraints}

Optimum reactor performance requires a symmetric core layout. In case of hexagonal fuel assemblies, the number of positions for fuel assemblies in a core layout of regular hexagonal shape is given by

$$
3 n(n-1)+1
$$

for $n$ rows of fuel assemblies. By removing three assemblies at each corner of the hexagon in order to obtain a more circular periphery the number of assemblies for $n$ rows of assemblies becomes

$$
3 n(n-1)-17 \quad n>3
$$

Since the control assemblies have to be arranged in a way that they span a hexagon, the number of positions for fuel assemblies is given by

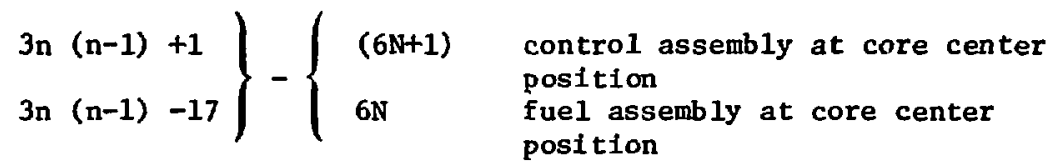

with $N$..... number of rings of control assemblies.

The variety of core arrangements can be increased slightly by arranging the control rods in a way that they span a triangle. This arrangement would tend to decrease the self-shielding of the control rods.

For zoned reactors, a series of one-dimensional burnup calculations can be performed to determine the reactor zone volumes and enrichments which promise optimum power peaking factors.

(b) physics constraints

A high fuel volume fraction is very desirable since the fuel volume fraction affects the reactor performance in several ways: an increase in the fuel volume fraction

1) increases the core convertion ratio and, therefore, reduces the control rod requirements and power swing in the core

2) increases the reactor breering ratio and, therefore, also the plutonium production

3) Increases the Doppler coefficlent 
Connected with an increase in fuel volume fraction is mostly an increase in the sodium void coefficient. However, ic is difficult today to assess the effect. of changes in the Doppler and sodium void coefficient on the fuel cycle cost performance. But it is known that a decrease in the number of control rods and a greater breeding ratio decrease the power cost.

A reduction in control rod requirements can also be obtained by shortening the refueling intervals. But for a fixed refueling scheduie an increase in the fuel volume fraction will always reduce the control rod requirements. A core conversion ratio close to or slightly above unity is the ultimate goal for reducing the burnup control rod requirements.

\section{(c) pressure drop constraint}

Maximum allowable nozzle to nozzle pressure drop is based on the expected 1imitations of a single-stage Impeller pump. Available information on single-stage sodfum pumps indicate that the maximum allowable pump head is $550 \mathrm{ft}$. of sodium.

At $900^{\circ} \mathrm{F}$ this corresponds to about 200 psi. This 1 imit can be relaxed only by adopting drastically new design concepts for the primary pump, e.g., a multistage pump. Considering this the maxlmum allowable pressure drop in the whole primary loop and the reactor the allowable system pressure drop is $11 \mathrm{mi}$ ted to 200 psi.

The pressure drop is the total primary system pressure drop and consists of contributions from the primary loop, the vessel internals, losses at assembly inlet and outlet and the pressure drop through the core.

The pressure drop limit constraints core height, fuel pitch-todiameter ratio and affects the pin design for a given spacer concept.

(d) sodium velocity constraint

Because of sodium corrosion and cavt tation the maximum sodium velocity through the core is limited. Experimental data on sodium corrosion, however, Indicate that the corrosion rate is not strongly dependent on sodium velocity. Cavitation starts at a certain velocity and severe damage can occur within a very short period of time if the maximum sodiun velocity stays above this critical velocity. It was shown in reference 1 that in case of a gridded fuel assembly the sodium velocity constraint has a significant impact on design. At the contact points between the dimples and the cladding the flow area is very restricted and high sodium velocities can occur.

\section{(e) burnup and fluence limic constraints}

The burnup limit aliows the designer to constrain the fuel pin diameter. This possibility exists because of the desire to come as close as possible to the peak burnup limit for an assembly when it is discharged. The assembly with the highest incremental burnup per cycle, briefly called peak assembly, should reach the peak burnup limit just before it is discharged from the reactor. Because of the discreteness of the problem, the average incremental burn, per cycle should be one-half, one-third, one- 
fourth, etc., of the peak burnup to assure optimum fuel utilization. Let us assume that the peak burnup is $100,000 \mathrm{MWd} / \mathrm{t}$. If the pin diameter (or more correctly pellet diameter) is selected such that the burnup during each cycle is $50,000 \mathrm{MWd} / t$, then this core can be operated on a two-batch refueling mode. On the other hand, if the incremental burnup over one cycle is $65,000 \mathrm{MWd} / \mathrm{t}$ then most of the fuel assemblies have to be discharged after one cycle already thus penalizing the performance severely. The proposed remedy in such a case is to increase the pellet diameter. This leads to a small increase in fissile inventory but leads also to an improved breeding ratio and reduced control requirements.

A correlation was derived between the smeared fuel pellet diameter d, the maximum neutron linear heat rating $r$, the peak assembly residence time $\mathrm{T}_{\mathrm{A}}$, the peak burnup limit Bupeak and the smeared fuel density $\rho$ (heavy metal density):

$$
d=2 \sqrt{\frac{r \cdot T_{A}}{\text { BU }^{\text {peak }} \cdot \rho \cdot \pi}} .
$$

Two assumptions were made in deriving the correlation. For one, it was assumed that the ratio of peak to average burnup is the sane as the ratio of peak to average power. For a one-batch core this assumption is correct. Only sma11 differences between these two peaking factors exist in multibatch cores.

The other assumption is that the power peak is stationary in the core. While this assumption is fairly accurate with respect to pin diameter selections in small LMFBR systems preliminary burnup calculations for the 5000 MNt LMFBR (reference 2) showed that the power peak will be in the outer core zone at the beginning of the cycle and at the core center at the end of the cycle. The reas on for this behavior is the difference in internal conversion ratio which is significantly above unity in the inner core zone while the outer core zone conversion ratio is below unity. This in itseif would not constitute a problem if the numerical values for the power peaking factors for the inner and outer core zone would be very close at any given time. But preliminary calculations showed that this might not always be the case. A further complication is expected due to the movement of control rods which affects the power shape and therefore power peaking significantly.

Often the design is constrained by a fluence limit and a burnup limit. It is then important to assure the compatibility of these two data.

\section{APPLICATION}

In an earlier study ( 1 ) the constrained nuclear design approach was utilized to select configuration and base design data for a $1000 \mathrm{MWe}$ oxide LMFBR core. Most recently the constrained nuclear design approach was utilized in an evaluation of the breeding performance of 5000 MWt LMFBR carbide cores (2) and in a carbide fuel pin optimization study (3).

In utilizing this approach assumptions have to be made with respect to the power peaking factor and the power split between core and blankets. Typical data obtained from simflar designs or results of one-dimensional 
calculations can be utillzed. The most significant result of the constrained design approach is the determination of the pin diameter. Figures 1 to 3 show the results of a pin optimization study for carbide fuel. The smeared pin diameter was determined as a function of the linear heat rating for different smeared fuel densitles, different burnup limits and different limits on fuel assembly residence time. For a given burnup limft, the most significant parameter for the determination of the fuel pin diameter is the limit on fuel assembly residence time. For a burnup limit of 100,000 $\mathrm{MWd} / \mathrm{t}$, a linear heat rating of $20 \mathrm{~kW} / \mathrm{ft}$ and a smeared fuel density of $85 \%$ T.D. the optimum smeared fuel pellet diameter for a single-batch core is below 0.2 inches. On the other hand a three-batch core operation requires a smeared fuel pellet diameter of more than 0.3 inches. This fuel pin optimization does not require the knowledge of actual fabrication cost. The diameters are selected such that the fabrication cost contribution to the fuel cycle cost will be minimum for whatever the actual cost are.

\section{v. CONCLUSIONS}

By imposing constraints on the design of a reactor it is possible to reduce the number of iterations between mechanical, thermal and nuclear design. These constraints come from the geometry of the reactor, material properties and our knowledge of the physics of reactors. In addition to reducing the number of design iterations this approach helps to identify key design parameters as well as key design constraints. In one study (1) the sodium arid velocity vas identified as one of the most important design constraints. In a study of the breeding potential of carbide cores, it was shown that the nuclear performance does not very strongly depend on the achievable linear heat rating. But the fuel assembly residence time was identified as a key design parameter.

The constrained nuclear design approach permits the reduction in the number of design iterations because it forces the reactor analyst to utilize existing knowledge of reactors. He can concentrate on the detalled analysis of a few realistic designs rather than the analysis of a large number of reactors with different volume fractions of fuel, coolant and structural material which do not exist in reality. The constrained nuclear design approaci emphasizes the interrelation between mechanical, thermal and nuclear design.

\section{REFERENCES}

1. W. P. Barthold, "Principles for the Selection of an LMFBR Core Configuration and Their Application to the Selection of a 1000 MWe LMFBR Oxide Core," WARD-116 (1969).

2. W. P. Barthold, J. Beite1, R. Turski, Private Communication

3. Reactor Development Program Progress Report, May 1974, ANL-RDP-28, p. 10.1-10.11 (1974). 


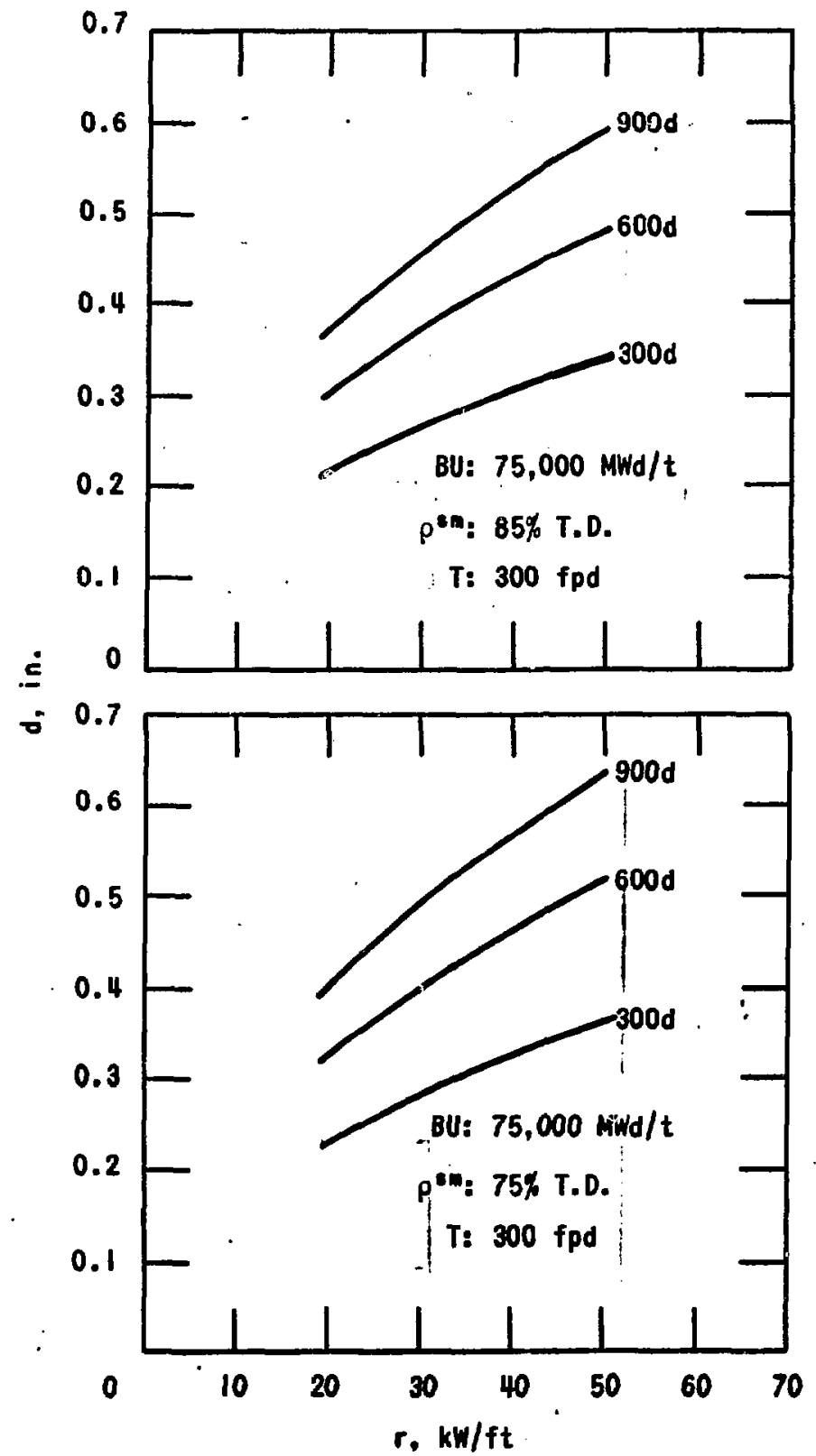

Fig. 1. Smeared Pellet Diameter as a Function of Maximum Nuclear Linear Heat Rating. for Peak Burnup Limit of $75,000 \mathrm{Mdd} / \mathrm{t}$ 


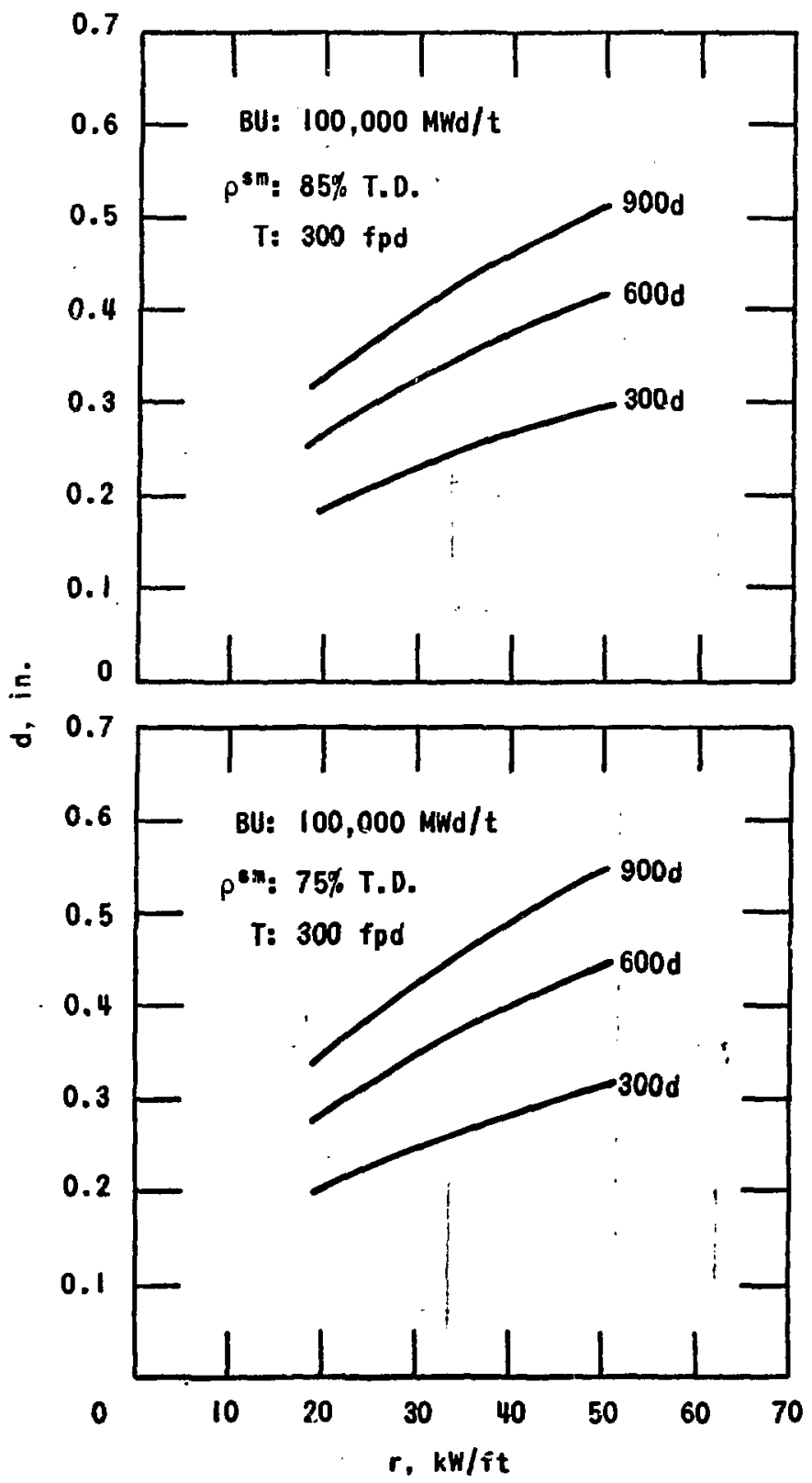

Fig. 2. Smeared Fellet Diameter as a Function of Maximum Nuclear Linear Heat Rating for Peak Burnup Limit of $100,000 \mathrm{Md} / \mathrm{t}$ 


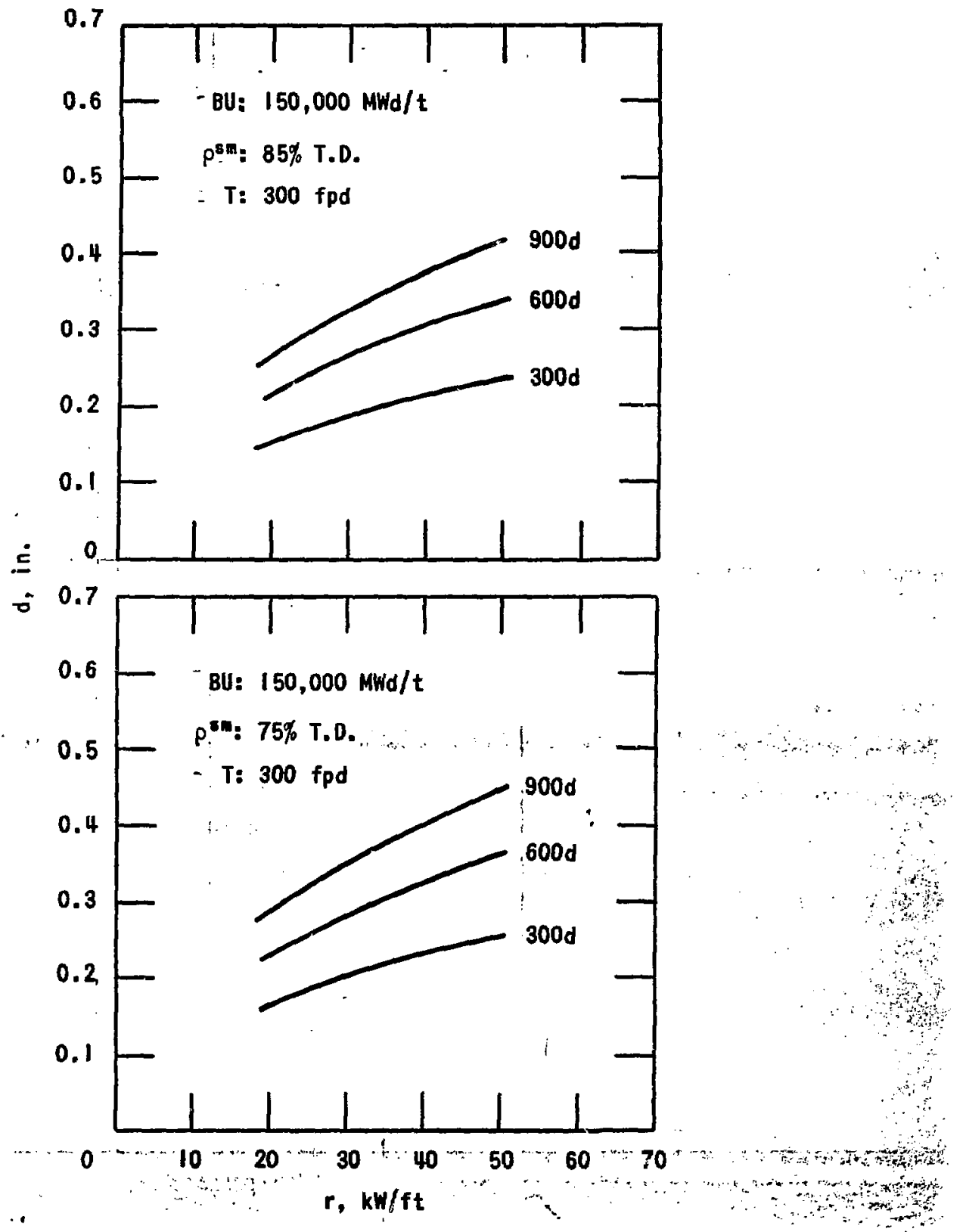

Fig. 3. Smeared Pellet Diameter as a Function of Maximum Nuclear Lineat Heat Rating for Peak Burnup Limit of $150,000 \mathrm{MNd} / \mathrm{t}$ 